\title{
Extraction of the Neutron Electric Form Factor from Measurements of Inclusive Double Spin Asymmetries
}

V. Sulkosky, ${ }^{1,2}$ G. Jin,${ }^{2}$ E. Long, ${ }^{3}$ Y.-W. Zhang, ${ }^{4}$ M. Mihovilovic, ${ }^{5}$ A. Kelleher, ${ }^{1}$ B. Anderson, ${ }^{6}$ D.W. Higinbotham, ${ }^{7}$, | S. Sirca,,${ }^{8,5}$ K. Allada,${ }^{7}$ J.R.M. Annand,${ }^{9}$ T. Averett, ${ }^{10}$ W. Bertozzi, ${ }^{1}$ W. Boeglin,,${ }^{11}$ P. Bradshaw, ${ }^{10}$ A. Camsonne, ${ }^{7}$ M. Canan, ${ }^{12}$ G.D. Cates,${ }^{2}$ C. Chen,${ }^{13}$ J.-P. Chen,${ }^{7}$ E. Chudakov, ${ }^{7}$ R. De Leo, ${ }^{14}$ X. Deng, ${ }^{2}$ A. Deur, ${ }^{7}$ C. Dutta,${ }^{15}$ L. El Fassi,${ }^{4}$ D. Flay, ${ }^{16}$ S. Frullani, ${ }^{17},{ }^{\dagger}$ F. Garibaldi, ${ }^{17}$ H. Gao,${ }^{18}$ S. Gilad,${ }^{1}$ R. Gilman,,${ }^{7}$ O. Glamazdin, ${ }^{19}$ S. Golge, ${ }^{12}$ J. Gomez, ${ }^{7}$ J.-O. Hansen, ${ }^{7}$ T. Holmstrom, ${ }^{20}$ J. Huang, ${ }^{1,21}$ H. Ibrahim, ${ }^{22}$ C.W. de Jager, ${ }^{7,2,}$ E. Jensen, ${ }^{23}$ X. Jiang, ${ }^{21}$ M. Jones, ${ }^{7}$ H. Kang, ${ }^{24}$ J. Katich, ${ }^{10}$ H.P. Khanal, ${ }^{11}$ P. King, ${ }^{25}$ W. Korsch, ${ }^{15}$ J. LeRose,${ }^{7}$ R. Lindgren,${ }^{2}$ H.-J. Lu, ${ }^{26}$ W. Luo,${ }^{27}$ P. Markowitz, ${ }^{11}$ D. Meekins, ${ }^{7}$ M. Meziane, ${ }^{10}$ R. Michaels,${ }^{7}$ B. Moffit,${ }^{7}$ P. Monaghan, ${ }^{13}$ N. Muangma,${ }^{1}$ S. Nanda, ${ }^{7}$ B.E. Norum,${ }^{2}$ K. Pan,${ }^{1}$ D. Parno ${ }^{28}$ E. Piasetzky, ${ }^{29}$ M. Posik, ${ }^{16}$ V. Punjabi, ${ }^{30}$ A.J.R. Puckett,${ }^{1}$ X. Qian, ${ }^{18}$ Y. Qiang, ${ }^{7}$ X. Qui, ${ }^{27}$ S. Riordan, ${ }^{2,31,32}$ A. Saha, ${ }^{7}$, ${ }^{7}$. Sawatzky, ${ }^{7}$ M. Shabestari, ${ }^{2}$ A. Shahinyan, ${ }^{33}$ B. Shoenrock, ${ }^{34}$ J. St. John, ${ }^{20}$ R. Subedi, ${ }^{35}$ W.A. Tobias, ${ }^{2}$ W. Tireman, ${ }^{34}$ G.M. Urciuoli, ${ }^{17}$ D. Wang, ${ }^{2}$ K. Wang, ${ }^{2}$ Y. Wang, ${ }^{36}$ J. Watson, ${ }^{6}$ B. Wojtsekhowski, ${ }^{7}$ Z. Ye ${ }^{13}$ X. Zhan, ${ }^{1}$ Y. Zhang, ${ }^{27}$ X. Zheng, ${ }^{2}$ B. Zhao, ${ }^{10}$ and L. Zhu ${ }^{13}$

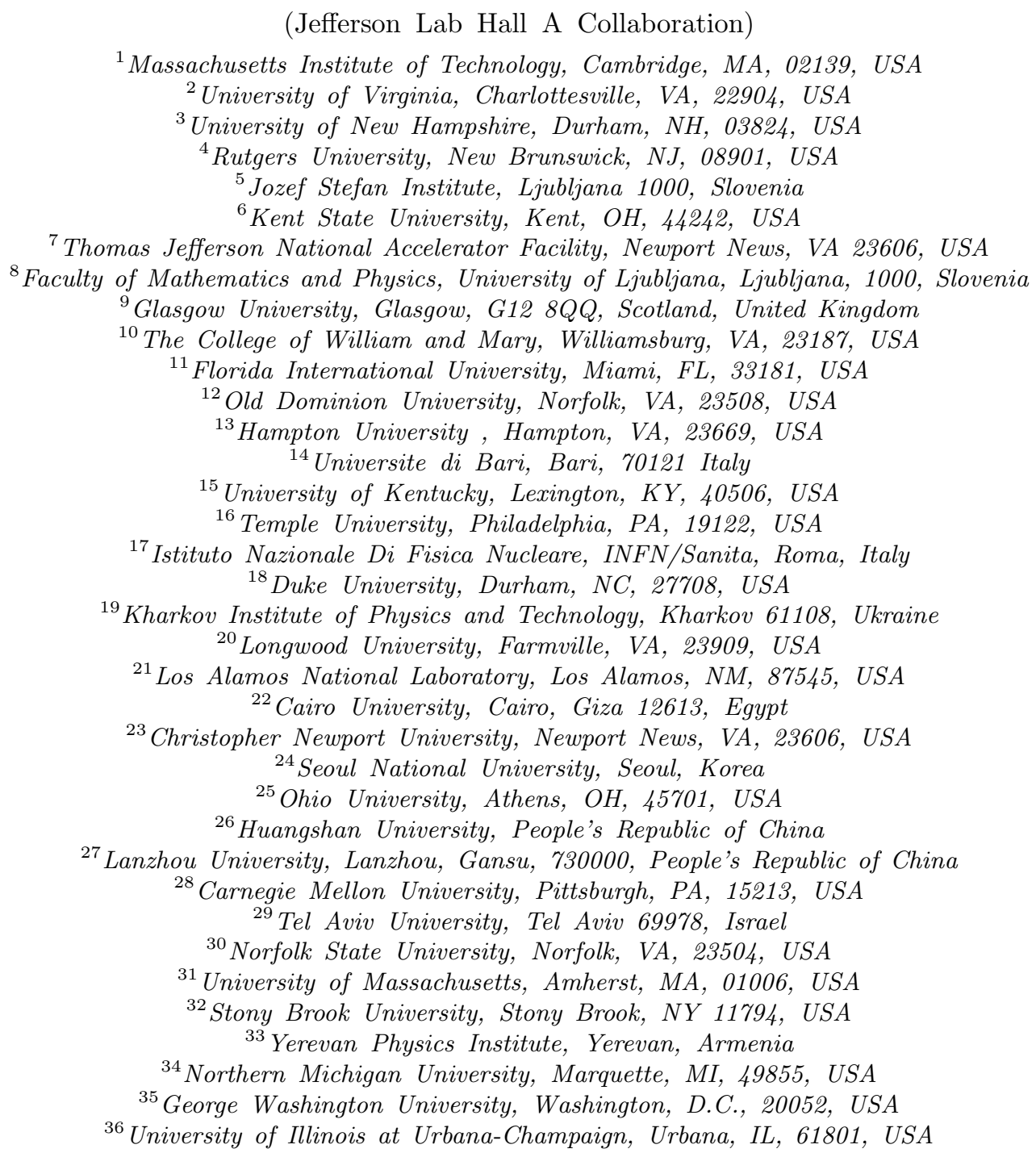

Background: Measurements of the neutron charge form factor, $G_{E}^{n}$, are challenging due to the fact that the neutron has no net charge. In addition, measurements of the neutron form factors must use nuclear targets which require accurately accounting for nuclear effects. Extracting $G_{E}^{n}$ with different targets and techniques provides an important test of our handling of these effects.

Purpose: The goal of the measurement was to use an inclusive asymmetry measurement technique to extract the neutron charge form factor at a four-momentum transfer of $1(\mathrm{GeV} / \mathrm{c})^{2}$. This technique has very different 
systematic uncertainties than traditional exclusive measurements and thus serves as an independent check of whether nuclear effects have been taken into account correctly.

Method: The inclusive quasi-elastic reaction ${ }^{3} \overrightarrow{\mathrm{He}}\left(\vec{e}, e^{\prime}\right)$ was measured at Jefferson Lab. The neutron electric form factor, $G_{E}^{n}$, was extracted at $Q^{2}=0.98(\mathrm{GeV} / \mathrm{c})^{2}$ from ratios of electron-polarization asymmetries measured for two orthogonal target spin orientations. This $Q^{2}$ is high enough that the sensitivity to $G_{E}^{n}$ is not overwhelmed by the neutron magnetic contribution, and yet low enough that explicit neutron detection is not required to suppress pion production.

Results: The neutron electric form factor, $G_{E}^{n}$, was determined to be $0.0414 \pm 0.0077$ (stat) \pm 0.0022 (syst); providing the first high precision inclusive extraction of the neutron's charge form factor.

Conclusions: The use of the inclusive quasi-elastic ${ }^{3} \overrightarrow{\mathrm{He}}\left(\vec{e}, e^{\prime}\right)$ with a four-momentum transfer near $1(\mathrm{GeV} / \mathrm{c})^{2}$ has been used to provide a unique measurement of $G_{E}^{n}$. This new result provides a systematically independent validation of the exclusive extraction technique results and implies that the nuclear corrections are understood. This is contrary to the proton form factor where asymmetry and cross-section measurements have been shown to have large systematic differences.

PACS numbers: 14.20.Dh, 13.40.Gp, 24.70.+s, 25.30.Bf

\section{INTRODUCTION}

Electromagnetic form factors describe the nucleon's static electromagnetic structure and provide insight into understanding nucleons in terms of their fundamental degrees of freedom. Of the four electromagnetic form factors of the proton and neutron $\left(G_{E}^{p}, G_{M}^{p}, G_{E}^{n}\right.$, and $G_{M}^{n}$ ), the measurement of $G_{E}^{n}$ is particularly challenging due to its small value and the difficulty in obtaining a high-density "pure" neutron target. Extractions of neutron form factors have relied on measurements on light nuclei, such as the deuteron or ${ }^{3} \mathrm{He}$, where the neutron is bound inside the nucleus. Experimental methods that provide access to $G_{E}^{n}$ include Rosenbluth separations from an unpolarized deuteron target [1, 2] and double-polarization measurements using either a polarized target [3 9] or an unpolarized target combined with a polarimeter to measure the polarization transfer to the recoiling neutron 10 13. At low four-momentumtransfer-squared, $Q^{2}$ from 0.1 to $0.2(\mathrm{GeV} / \mathrm{c})^{2}$, inclusive quasi-elastic scattering from a polarized ${ }^{3} \mathrm{He}$ target was also tried [14, 15]. However, these early measurements yielded statistical uncertainties comparable with the extracted quantity; the sources of theoretical uncertainties were not investigated. In a later measurement, better statistical precision was obtained, and an extensive analysis of the systematic uncertainties was performed [16]. In that analysis, the large variation in the asymmetry predictions revealed a large model uncertainty at low $Q^{2}$. The authors of that paper suggested that the extraction would be likely to succeed at higher $Q^{2}$. We report in this paper an extraction of $G_{E}^{n}$ at $Q^{2}=0.98(\mathrm{GeV} / \mathrm{c})^{2}$ from measurements of the ratios of two asymmetries in the ${ }^{3} \overrightarrow{\mathrm{He}}\left(\vec{e}, e^{\prime}\right)$ reaction where the ${ }^{3} \mathrm{He}$ spin vectors aligned parallel and orthogonal to the electron beam direction.

\footnotetext{
* Corresponding author: doug@jlab.org

$\dagger$ Deceased
}

\section{METHODS}

The measurements were performed at Jefferson Lab in experimental Hall A. A longitudinally polarized electron beam of $3.606 \mathrm{GeV}$ was scattered from a gaseous polarized ${ }^{3} \mathrm{He}$ target. The beam current was between $10 \mu \mathrm{A}$ and $15 \mu \mathrm{A}$, and the helicity of the beam was flipped at a frequency of $30 \mathrm{~Hz}$. During the experiment, the beam charge asymmetry was minimized by a beam charge feedback system [17] and was controlled to be less than 100 parts per million (ppm) per 20-30 min time period. Interruptions of the beam were found to have negligible effects on the asymmetry. As a dedicated beam polarization measurement in Hall A was not conducted during the period the data were taken, the average beam polarization was determined from measurements taken in Hall B with a Møller polarimeter to be $(82 \pm 2.5) \%$ [18.

A polarized ${ }^{3} \mathrm{He}$ target was used as an effective polarized neutron target. The target, made of aluminosilicate glass, consisted of a pumping chamber and a target cell. The spherical pumping chamber was located above the cylindrical target cell and was connected to the target cell by a transfer tube. The ${ }^{3} \mathrm{He}$ nuclei were polarized via spin-exchange optical pumping of a $\mathrm{Rb}-\mathrm{K}$ mixture [19]. The vapor of the alkali mixture was polarized in the pumping chamber, where the spin exchange with the ${ }^{3} \mathrm{He}$ nuclei occurred. The $40-\mathrm{cm}$-long target cell contained ${ }^{3} \mathrm{He}$ gas at $12 \mathrm{~atm}$, which provided a luminosity of $10^{36} \mathrm{~cm}^{-2} \mathrm{~s}^{-1}$. A small amount $(\simeq 2 \%$ in number density) of $\mathrm{N}_{2}$ gas was added to the target cell to absorb unwanted photons emitted from the Rb de-excitation process. With the aid of spectrally narrowed lasers that increase the light absorption efficiency [20, a significant improvement in target polarization was achieved compared to previous experiments with similar targets. The polarization of the cell was measured every 6 hours using nuclear magnetic resonance, calibrated using electron paramagnetic resonance 21] polarimetry. An average inbeam target polarization of $(50.2 \pm 2.5) \%$ was achieved. Additionally, a reference cell that could be filled with either ${ }^{3} \mathrm{He}, \mathrm{N}_{2}$, or $\mathrm{H}_{2}$ gas, was used to determine the 
dilution factors for the unpolarized material in the cell.

The scattered electrons were detected in the Right High Resolution Spectrometer (RHRS) 22. The RHRS was located at a forward angle of $17^{\circ}$ with respect to the incident beam direction and its central momentum was set to $3.086 \mathrm{GeV} / \mathrm{c}$. Thus, the momentum transfer to the target $(\vec{q})$ by an electron scattered into the center of the RHRS acceptance was pointing at an angle of $56^{\circ}$ with respect to the incident beam direction. Scattered electrons traveled through the RHRS by passing through a pair of superconducting $\cos (2 \theta)$ quadrupoles, a $6.6-\mathrm{m}-$ long dipole magnet, and a third superconducting $\cos (2 \theta)$ quadrupole. The detector package included: a pair of vertical drift chambers to determine the trajectory of a particle; two scintillator planes to provide the trigger; and a gas Cherenkov detector combined with a lead-glass electromagnetic calorimeter to separate electrons and pions. The spectrometer has a solid angle acceptance of $6 \mathrm{msr}$ and a momentum acceptance of $\pm 4.5 \%$. The spectrometer optics calibration resulted in the following resolutions: $6 \mathrm{~mm}$ in the vertex position along the beamline, $2 \times 10^{-4}$ in relative momentum, $1.5 \mathrm{mrad}$ in the out-ofplane angle and $0.5 \mathrm{mrad}$ in the in-plane angle.

During the experiment, two sets of Helmholtz coils were used to align the ${ }^{3} \mathrm{He}$ spin vector either parallel or perpendicular to the beam direction. This enabled us to measure independently the asymmetries, $A_{\|}$and $A_{\perp}$, where the subscripts indicate the orientation of the ${ }^{3} \mathrm{He}$ spin vector with respect to the beam and in the horizontal lab-frame plane. The experimental physics asymmetries were calculated by

$$
A=\frac{1}{P_{e} P_{t} f_{\mathrm{N}_{2}}}\left(\frac{Y^{+}-Y^{-}}{Y^{+}+Y^{-}}\right)
$$

where $Y^{ \pm}=\frac{N^{ \pm}}{Q^{ \pm} \cdot L T^{ \pm}}$represents the normalized yield for beam helicity $\pm 1, N^{ \pm}$is the number of detected scattered electrons, $Q^{ \pm}$is the accumulated charge, and $L T^{ \pm}$is the data acquisition live-time. $P_{e}$ and $P_{t}$ are the beam and target polarizations, respectively, and $f_{\mathrm{N}_{2}}$ is the dilution factor due to the admixture of $\mathrm{N}_{2}$ gas in the target cell. The dedicated $\mathrm{N}_{2}$ reference cell data were used to determine $f_{\mathrm{N}_{2}}=(95 \pm 2) \%$. The measured asymmetries were calculated near the quasi-elastic peak for values of the Bjorken scaling variable $x_{\mathrm{B}}=Q^{2} /(2 M \omega)$ in the range $0.9<x_{\mathrm{B}}<1.1$, where $M$ is the mass of the nucleon and $\omega=E-E^{\prime}$, where $E\left(E^{\prime}\right)$ is the incident (scattered) electron energy.

Radiative corrections were calculated based on the formalism of Mo and Tsai 23] with the program RADCOR.F [24]. This code was updated to use the peaking approximation of Stein et al. 25] and can perform both external and internal corrections for unpolarized and polarized cross sections. For the polarized cross sections, the relative uncertainty of the radiative corrections was estimated to be $20 \%$ and up to $40 \%$, when extrapolation from the model is involved [24]. The data from Ref. [26] were used to build a model for the two helicity states and extrapolated to the kinematics of this paper. The model cross sections were then incorporated into the radiative correction procedure. The size of the corrections for the asymmetries varied from $8 \%$ to $14 \%$ across the $x_{\mathrm{B}}$ acceptance. Due to the assumptions used in building the model and the extrapolation, a conservative relative uncertainty of $50 \%$ was chosen for the radiative corrections, which results in a relative uncertainty of about $5 \%$ for the corrected asymmetries. Since these measurements were done at a moderate $Q^{2}$ and epsilon near unity, twophoton effects corrections should be small and thus have been neglected.

\section{RESULTS}

The $A_{\|}$and $A_{\perp}$ inclusive ${ }^{3} \mathrm{He}$ asymmetries averaged over the spectrometer acceptance and after applying radiative corrections are shown in Fig. 1 with their values provided in Table II The inner error bars represent the statistical uncertainties; the outer error bars show the combined statistical and systematic uncertainties. For the parallel asymmetry $\left(A_{\|}\right)$, the statistical precision overwhelms the systematic uncertainty and, hence, the total error bar cannot be easily distinguished from the statistical error bar. The dominant experimental systematic uncertainties for the measured asymmetries are the uncertainty in the radiative corrections (5\%), the target polarization (5\%), the beam polarization $(3 \%)$ and the dilution factor $(2 \%)$, where all the uncertainties are relative to the asymmetry. The uncertainty due to inelastic backgrounds was not considered, since the statistical uncertainties dominate the total uncertainty. Within the statistical uncertainties, $A_{\|}$is almost constant across the chosen $x_{\mathrm{B}}$ range, whereas $A_{\perp}$ exhibits a slight linear decrease with increasing $x_{\mathrm{B}}$.

TABLE I. Parallel $\left(A_{\|}\right)$and transverse $\left(A_{\perp}\right)$ asymmetries near the quasi-elastic peak versus $x_{\mathrm{B}}$. The format for the asymmetries follows central value \pm statistical uncertainty \pm systematic uncertainty.

\begin{tabular}{lcc}
\hline$x_{\mathrm{B}}$ & $A_{\perp}(\%)$ & $A_{\|}(\%)$ \\
\hline 0.925 & $3.45 \pm 0.19 \pm 0.23$ & $-0.35 \pm 0.17 \pm 0.02$ \\
0.975 & $3.29 \pm 0.20 \pm 0.23$ & $-0.66 \pm 0.18 \pm 0.05$ \\
1.025 & $2.97 \pm 0.22 \pm 0.21$ & $-0.55 \pm 0.20 \pm 0.04$ \\
1.075 & $2.86 \pm 0.26 \pm 0.20$ & $-0.64 \pm 0.23 \pm 0.05$ \\
\hline
\end{tabular}

To relate the measured asymmetries to $G_{E}^{n}$ we used the formalism of Donnelly and Raskin [27] for scattering from a free spin- $1 / 2$ particle. The asymmetry for the ${ }^{3} \overrightarrow{\mathrm{He}}\left(\vec{e}, e^{\prime}\right)$ reaction near the quasi-elastic peak can be written in terms of ${ }^{3} \mathrm{He}$ response functions as the ratio of the spin-averaged $(\Sigma)$ and polarization $(\Delta)$ cross sections: 


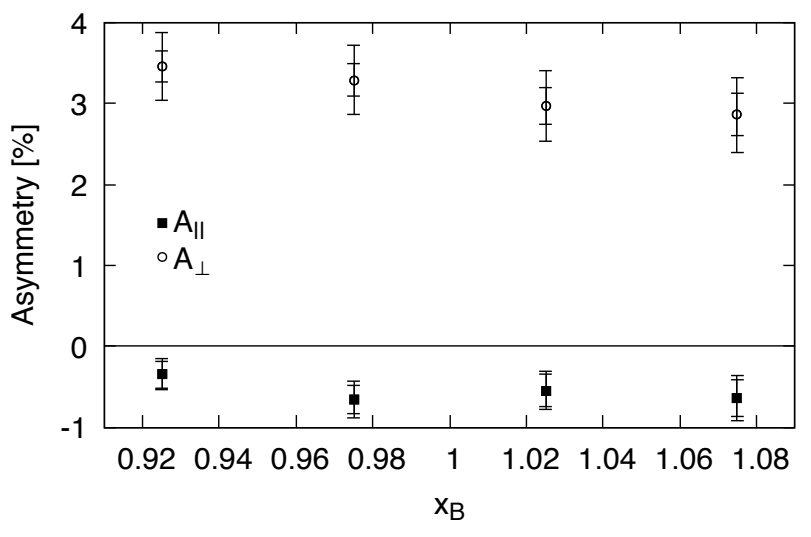

FIG. 1. Inclusive asymmetries from the ${ }^{3} \overrightarrow{\mathrm{He}}\left(\vec{e}, e^{\prime}\right)$ reaction with the target spin parallel, $\left(A_{\|}\right)$, and transverse, $\left(A_{\perp}\right)$, to the electron beam direction asymmetries near the quasielastic peak versus $x_{B}$. The inner (outer) error bars represent the statistical (statistical plus systematic) uncertainties.

$$
\begin{aligned}
& A\left(\theta^{*}, \phi^{*}\right)=\frac{\Delta\left(\theta^{*}, \phi^{*}\right)}{\Sigma\left(\theta^{*}, \phi^{*}\right)} \\
& =-\frac{\cos \theta^{*} v_{T^{\prime}} R_{T^{\prime}}^{3 \mathrm{He}}+\sin \theta^{*} \cos \phi^{*} v_{T L^{\prime}} R_{T L^{\prime}}^{3} \mathrm{He}}{v_{L} R_{L}^{3} \mathrm{He}}+v_{T} R_{T}^{3} \mathrm{He}
\end{aligned}
$$

where $R_{T^{\prime}\left(T L^{\prime}\right)}^{3 \mathrm{He}}$ are the ${ }^{3} \mathrm{He}$ polarized transverse (transverse-longitudinal) response functions, $R_{T(L)}^{3 \mathrm{He}}$ are the ${ }^{3} \mathrm{He}$ unpolarized transverse (longitudinal) response functions, and the $v$ 's are kinematic factors which are independent of beam and target polarizations. $\theta^{*}$ and $\phi^{*}$ are, respectively, the polar and azimuthal angles of the target polarization vector with respect to the threemomentum transfer $\vec{q}$. Thus, asymmetries measured with the target oriented parallel (perpendicular) to the electron beam correspond to $\theta^{*}=56^{\circ}$ and $\phi^{*}=0^{\circ}$ $\left(\theta^{*}=34^{\circ}\right.$ and $\left.\phi^{*}=180^{\circ}\right)$.

Following the Plane-Wave-Impulse-Approximation (PWIA) calculation by Kievsky et al. 28, the polarized ${ }^{3} \mathrm{He}$ transverse (transverse-longitudinal) response functions $R_{T^{\prime}\left(T L^{\prime}\right)}^{3 \mathrm{He}}$ near the quasi-elastic peak are written as

$$
\begin{gathered}
R_{T^{\prime}}^{3} \mathrm{He}=\frac{Q^{2}}{2 q M}\left(2\left[G_{M}^{p}\right]^{2} H_{T^{\prime}}^{p}+\left[G_{M}^{n}\right]^{2} H_{T^{\prime}}^{n}\right), \\
R_{T L^{\prime}}^{3 \mathrm{He}}=-\sqrt{2}\left(2 G_{M}^{p} G_{E}^{p} H_{T L^{\prime}}^{p}+G_{M}^{n} G_{E}^{n} H_{T L^{\prime}}^{n}\right),
\end{gathered}
$$

where the $H_{S}^{p(n)}$ represent the proton (neutron) contribution to the response functions with $S=T^{\prime}$ or $T L^{\prime}$. The proton form factors, $G_{E}^{p}$ and $G_{M}^{p}$, as well as the neutron magnetic form factor $G_{M}^{n}$, were constrained by the world data. The values of $H_{S}^{n(p)}$ were calculated in Ref. [28] using models for the nucleon polarizations and momentum distributions in the ${ }^{3} \mathrm{He}$ nuclei. These values are almost constant over a wide range of $Q^{2}$. Thus, by measuring $A$ for two sets of $\left(\theta^{*}, \phi^{*}\right)$ at electron scattering angles and scattered electron momenta spanning the acceptance of the RHRS subject to the constraint that $0.9<x_{\mathrm{B}}<1.1$, we obtain two linearly independent equations. The dependence on the ${ }^{3} \mathrm{He}$ unpolarized response functions can be removed by taking the ratio of $A\left(\theta^{*}, \phi^{*}\right)$ for two sets of $\left(\theta^{*}, \phi^{*}\right)$ :

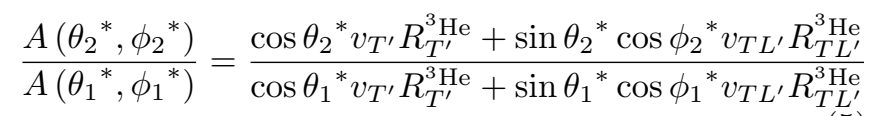

which can be solved for $G_{E}^{n}$.

The uncertainties in the ratios of asymmetries were dominated by the statistical uncertainty (18.5\%) in the values of $A_{\|}$. In these ratios, the absolute values of corrections such as the beam and target polarizations cancel to first order and only their relative changes during the measurement contribute to the uncertainty. We estimate that the uncertainties in the asymmetry ratio from the beam and target polarizations are $\simeq 3 \%$ and $\simeq 1 \%$, respectively. Similarly, the dilution factors cancel. The radiative corrections are correlated for the two measured asymmetries so that they also mostly cancel in the ratio. When the radiative corrections are varied within the uncertainties, we found the ratios of the asymmetries change by $\ll 1 \%$; however, due to the assumptions made in their determination, we have taken $1 \%$ to be a conservative estimate of the uncertainty from this source. Finally, the measurement of the asymmetries is sensitive to the target polarization angle $\theta^{*}$ that has an uncertainty of $\pm 0.3^{\circ}$. This results in a $3 \%$ uncertainty in the ratio. We estimate the total experimental systematic uncertainty to be $4.5 \%$ for the ratios of the asymmetries.

The discussion up to this point has been based on the PWIA framework. Corrections to this approximation must be considered. The effects of final state interactions (FSI) were examined and found to decrease significantly with increasing $Q^{2}[29,30$. The PWIA calculation mentioned previously [28] was used in earlier determinations of $G_{M}^{n}$ in the range $Q^{2}=0.1-0.6(\mathrm{GeV} / \mathrm{c})^{2}$ from measurements of the $A_{T^{\prime}}$ asymmetry made at Jefferson Lab 31. The effects of FSI were greatly reduced above $Q^{2}$ of $0.5(\mathrm{GeV} / \mathrm{c})^{2}$, and for $Q^{2} \geq 1(\mathrm{GeV} / \mathrm{c})^{2}$ FSI corrections are expected to fall as $Q^{-4}$. Corrections for meson-exchange currents (MEC) are expected to be negligible at the quasi-elastic peak 32 and to decrease exponentially as $Q^{2}$ increases, based on the observation in Ref. 31] as well as the calculations of Golak 33.

Within the context of PWIA, inclusion of the off-shell nature of the struck nucleon into the calculation of the electron-nucleon cross section requires a model of the nucleon current. In particular, a model for the contribution of the anomalous magnetic moment of the struck nucleon must be chosen. The CC1 and CC2 prescrip- 
tions of De Forest [34] for off-shell cross sections were used to obtain the ${ }^{3} \mathrm{He}$ responses. In these two prescriptions the off-shell effects are incorporated into the electron kinematics using different approaches as outlined in ref. 34. In the $\mathrm{CC} 1$ prescription the four-momentum transfer is determined solely by the electron kinematics. In the $\mathrm{CC} 2$ prescription the three-momentum transfer, $\vec{q}$, is determined by the electron kinematics and the energy transfer from the final energy and initial momentum of the struck nucleon. It is to be noted that in both cases energy-momentum and current conversation are violated as in both cases the nucleons are treated as free particles. PWIA calculations using these forms provide good agreement with the unpolarized ${ }^{3} \mathrm{He}$ response functions [28]. For the polarized responses, both prescriptions provide essentially the same result for $R_{T L^{\prime}}$, while the results for $R_{T^{\prime}}$ in general differ less than $2 \%$ over the range of $0.1 \leq Q^{2} \leq 2(\mathrm{GeV} / \mathrm{c})^{2}$. Due to these differences, only the results from $\mathrm{CC} 1$ were reported in Ref. [28. Other prescriptions are available [35] but were not considered.

When $x_{\mathrm{B}}$ is near 1 , the struck nucleon is almost at rest before absorbing the virtual photon. After absorbing the photon, it has a momentum almost equal to that of the virtual photon. In the kinematics of this paper, the struck nucleon has a relativistic kinetic energy. Hence, the inclusion of relativistic effects in the theoretical calculations is essential. The uncertainty due to these effects was estimated in Ref. [32. A comparison was made within the Virtual Nucleon and Light Cone approximations, which are different treatments of the relativistic motion of bound nucleons as well as electromagnetic currents. The difference between the predictions made using these two approximations was found to be $1.2 \%$ at $Q^{2} \simeq 1(\mathrm{GeV} / \mathrm{c})^{2}$.

Parameterizations of the three undetermined electromagnetic form factors were used as inputs to calculate an asymmetry at each kinematic point over the measured angular and momentum acceptance of the RHRS. For $G_{M}^{n}$ the high precision data from Ref. [36] were used, and the values for $G_{E}^{p}$ and $G_{M}^{p}$ were provided by Refs. [37, 38], which were extracted after applying the two-photon exchange corrections as done in 39. The values and uncertainties for the form factors used in the extraction at the central value of $Q^{2}$ are presented in Table II. Taking into account the correlations between $G_{E}^{p}$ and $G_{M}^{p}$, these uncertainties in the form factors lead to an uncertainty of $1.4 \%$ in the extracted value of $G_{E}^{n}$. The extraction of $G_{E}^{n}$ is not limited by the uncertainties on the individual form factors. Examining Eq. (5) reveals that the proton contributions to the response functions are suppressed in ${ }^{3} \mathrm{He}$, and hence, their uncertainties in the extraction of $G_{E}^{n}$ are also suppressed. On the other hand, as $Q^{2}$ increases the uncertainty on $G_{M}^{n}(2.1-2.6 \%)$ becomes important at $Q^{2}=2.6(\mathrm{GeV} / \mathrm{c})^{2}$. Finally, the uncertainty on $G_{E}^{p}$ grows linearly with $Q^{2}$ and adds an equal uncertainty in the extraction of $G_{E}^{n}$ at this $Q^{2}$.

Using Eq. (5), the central value of $G_{E}^{n}$ was varied to fit the calculated ratio of asymmetries to the experimentally
TABLE II. The values and uncertainties for the form factors used in the extraction at $Q^{2}=0.98(\mathrm{GeV} / \mathrm{c})^{2}$. The column $\delta G_{E}^{n}$ provides the contributions to the systematic uncertainty of $G_{E}^{n}$ from the input form factors to Eq. (2).

\begin{tabular}{lcc}
\hline Form Factor & Value & $\delta G_{E}^{n}$ \\
\hline$G_{E}^{p} / G_{D}$ & $0.9413 \pm 0.0094$ & $3.0 \times 10^{-4}$ \\
$\mu_{p} G_{M}^{p} / G_{D}$ & $1.0456 \pm 0.0104$ & $2.5 \times 10^{-4}$ \\
$\mu_{n} G_{M}^{n} / G_{D}$ & $0.9953 \pm 0.0225$ & $1.9 \times 10^{-4}$ \\
\hline
\end{tabular}

measured ratios. The value for $G_{E}^{n}$ extracted at $Q^{2}=$ $0.98(\mathrm{GeV} / \mathrm{c})^{2}$ is

$$
G_{E}^{n}=0.0414 \pm 0.0077 \pm 0.0022,
$$

where the first (second) uncertainty is statistical (systematic). In Fig. 2, the present result for $G_{E}^{n}$ is shown as the solid square along with selected world data and parametrizations. The extracted result is consistent with the world data, showing the feasibility of this method for values of $Q^{2}$ larger than $0.8(\mathrm{GeV} / \mathrm{c})^{2}$. It should be noted that the present data were acquired in only 2.5 days of running. As the extraction of $G_{E}^{n}$ was not the principal focus of the measurements, the running time was divided evenly between the two target polarization orientations: parallel to the electron beam and perpendicular to the beam. Had the division of running time been optimized, with $90 \%(10 \%)$ of the time allocated to the parallel (perpendicular) orientation, the statistical uncertainty on $G_{E}^{n}$ would be reduced from 0.0077 to 0.0026 .

\section{SUMMARY}

In summary, an extraction of $G_{E}^{n}$ from inclusive polarized ${ }^{3} \overrightarrow{\mathrm{He}}\left(\vec{e}, e^{\prime}\right)$ quasi-elastic asymmetry measurements was presented. This method of forming the ratio of inclusive asymmetries provides an important independent check of other measurements and has several advantages. Firstly, the systematic uncertainties associated with neutron detection 8 are avoided. Secondly, the sensitivity to certain unavoidable systematic errors (beam and target polarizations, dilution factors and radiative corrections) are greatly reduced due to first-order cancellations in the ratio of asymmetries. The final result at $Q^{2}=0.98(\mathrm{GeV} / \mathrm{c})^{2}$ of $G_{E}^{n}=0.0414 \pm 0.0077 \pm 0.0019$ was found to be consistent with other extraction techniques. This is in contrast to the proton, where at this same $Q^{2}$, systematic differences between form factor extraction techniques were revealed [4].

We thank the Jefferson Lab Physics and Accelerator Divisions. This work was supported in part by the U.S. National Science Foundation and by the U.S. Department of Energy. It is supported by DOE contract No. 


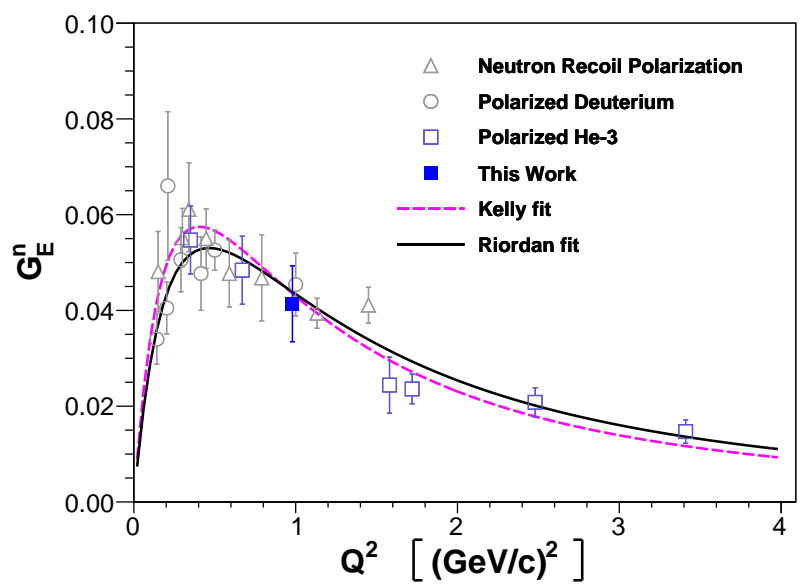

FIG. 2. The $G_{E}^{n}$ value extracted from this experiment (solid square) and selected published data: open triangles [10 13], open circles [3 5, open squares [6] 9] and parametrizations: Riordan et al. 8] and Kelly [0]. In regards to the polarized ${ }^{3} \mathrm{He}$ points, the solid square is from the inclusive reaction, whereas the open squares represent extracted results from experiments in which the neutron was tagged. The error bars show the statistical and the systematic uncertainties added in quadrature.
DE-AC05-06OR23177, under which Jefferson Science Associates (JSA), LLC, operates the Thomas Jefferson National Accelerator Facility.
[1] S. Galster, H. Klein, J. Moritz, K. H. Schmidt, D. Wegener, and J. Bleckwenn, Nucl. Phys. B32, 221 (1971).

[2] S. Platchkov et al., Nucl. Phys. A510, 740 (1990).

[3] I. Passchier et al., Phys. Rev. Lett. 82, 4988 (1999), arXiv:nucl-ex/9907012 [nucl-ex]

[4] G. Warren et al. (Jefferson Lab E93-026), Phys. Rev. Lett. 92, 042301 (2004), arXiv:nucl-ex/0308021 [nucl-ex].

[5] E. Geis et al. (BLAST), Phys. Rev. Lett. 101, 042501 (2008), arXiv:0803.3827 [nucl-ex].

[6] J. Becker et al., Eur. Phys. J. A6, 329 (1999).

[7] J. Bermuth et al., Phys. Lett. B564, 199 (2003), arXiv:nucl-ex/0303015 [nucl-ex]

[8] S. Riordan et al., Phys. Rev. Lett. 105, 262302 (2010), arXiv:1008.1738 [nucl-ex].

[9] B. S. Schlimme et al., Phys. Rev. Lett. 111, 132504 (2013), arXiv:1307.7361 [nucl-ex]

[10] C. Herberg et al., Eur. Phys. J. A5, 131 (1999),

[11] M. Ostrick et al., Phys. Rev. Lett. 83, 276 (1999)

[12] D. I. Glazier et al., Eur. Phys. J. A24, 101 (2005), arXiv:nucl-ex/0410026 [nucl-ex]

[13] B. Plaster et al. (Jefferson Laboratory E93-038), Phys. Rev. C73, 025205 (2006), arXiv:nucl-ex/0511025 [nuclex],

[14] C. E. Jones-Woodward et al., Phys. Rev. C44, R571 (1991)

[15] A. K. Thompson et al., Phys. Rev. Lett. 68, 2901 (1992)

[16] J. O. Hansen et al., Phys. Rev. Lett. 74, 654 (1995).

[17] D. Androic et al. (G0), Nucl. Instrum. Meth. A646, 59 (2011), arXiv:1103.0761 [nucl-ex]

[18] G. Jin, Ph.D. thesis, University of Virginia (2011).

[19] E. Babcock, I. Nelson, S. Kadlecek, B. Driehuys, L. W. Anderson, F. W. Hersman, and T. G. Walker, Phys.
Rev. Lett. 91, 123003 (2003).

[20] J. Singh, P. A. M. Dolph, W. A. Tobias, T. D. Averett, A. Kelleher, K. E. Mooney, V. V. Nelyubin, Y. Wang, Y. Zheng, and G. D. Cates, Phys. Rev. C91, 055205 (2015), arXiv:1309.4004 [physics.atom-ph]

21] M. V. Romalis et al., Polarized He-3 beams and gas targets and their application. Proceedings, 7th RCNP International Workshop, HELION'97, Kobe, Japan, January 20-24, 1997, Nucl. Instrum. Meth. A402, 260 (1998)

[22] J. Alcorn et al., Nucl. Instrum. Meth. A522, 294 (2004).

[23] L. W. Mo and Y.-S. Tsai, Rev. Mod. Phys. 41, 205 (1969)

[24] K. Slifer, Ph.D. thesis, Temple University (2004).

[25] S. Stein, W. B. Atwood, E. D. Bloom, R. L. Cottrell, H. C. DeStaebler, C. L. Jordan, H. Piel, C. Y. Prescott, R. Siemann, and R. E. Taylor, Phys. Rev. D12, 1884 (1975)

[26] K. Slifer et al. (E94010), Phys. Rev. Lett. 101, 022303 (2008), arXiv:0803.2267 [nucl-ex]

[27] T. W. Donnelly and A. S. Raskin, Annals Phys. 169, 247 (1986)

[28] A. Kievsky, E. Pace, G. Salme, and M. Viviani, Phys. Rev. C56, 64 (1997), arXiv:nucl-th/9704050 [nucl-th].

[29] E. Pace, G. Salme, and G. B. West, Phys. Lett. B273, 205 (1991)

[30] O. Benhar, Phys. Rev. Lett. 83, 3130 (1999), arXiv:nuclth/9908086 [nucl-th]

[31] W. Xu et al. (Jefferson Lab E95-001), Phys. Rev. C67, 012201 (2003), arXiv:nucl-ex/0208007 [nucl-ex].

[32] M. Sargsian, private communication (2012).

[33] J. Golak, G. Ziemer, H. Kamada, H. Witala, and W. Gloeckle, Phys. Rev. C63, 034006 (2001), arXiv:nucl- 
th/0008008 [nucl-th]

[34] T. De Forest, Nucl. Phys. A392, 232 (1983).

[35] J. A. Caballero, T. W. Donnelly, and G. I. Poulis, Nucl. Phys. A555, 709 (1993).

[36] J. Lachniet et al. (CLAS), Phys. Rev. Lett. 102, 192001 (2009), arXiv:0811.1716 [nucl-ex]

[37] S. Venkat, J. Arrington, G. A. Miller, and X. Zhan, Phys.
Rev. C83, 015203 (2011), arXiv:1010.3629 [nucl-th] [38] J. Arrington, private communication (2012).

[39] J. Arrington, W. Melnitchouk, and J. A. Tjon, Phys. Rev. C76, 035205 (2007), arXiv:0707.1861 [nucl-ex]

[40] J. J. Kelly, Phys. Rev. C70, 068202 (2004).

[41] M. K. Jones et al. (Jefferson Lab Hall A), Phys. Rev. Lett. 84, 1398 (2000), arXiv:nucl-ex/9910005 [nucl-ex]. 